\title{
RINGS WITH TRIVIAL FML-INVARIANT
}

\author{
DANIEL DAIGLE
}

\begin{abstract}
Let $\mathbf{k}$ be a field of characteristic zero and $B$ a commutative integral domain that is also a finitely generated $\mathbf{k}$-algebra. It is well known that if $\mathbf{k}$ is algebraically closed and the "Field MakarLimanov" invariant FML $(B)$ is equal to $\mathbf{k}$, then $B$ is unirational over $\mathbf{k}$. This article shows that, when $\mathbf{k}$ is not assumed to be algebraically closed, the condition $\operatorname{FML}(B)=\mathbf{k}$ implies that there exists a nonempty Zariski-open subset $U$ of Spec $B$ with the following property: for each prime ideal $\mathfrak{p} \in U$, the $\kappa(\mathfrak{p})$-algebra $\kappa(\mathfrak{p}) \otimes_{\mathbf{k}} B$ can be embedded in a polynomial ring in $n$ variables over $\kappa(\mathfrak{p})$, where $n=\operatorname{dim} B$ and $\kappa(\mathfrak{p})=B_{\mathfrak{p}} / \mathfrak{p} B_{\mathfrak{p}}$.
\end{abstract}

\section{INTRODUCTION}

In this article, the word ring means commutative ring with a unity. By a domain, we mean a commutative integral domain. If $A$ is a domain then $\operatorname{Frac}(A)$ is its field of fractions. If $\mathbf{k}$ is a field, then a $\mathbf{k}$-domain is a domain that is also a $\mathbf{k}$-algebra; by an affine $\mathbf{k}$-domain we mean a $\mathbf{k}$-domain that is finitely generated as a $\mathbf{k}$-algebra.

If $B$ is a ring, a derivation $D: B \rightarrow B$ is locally nilpotent if for each $x \in B$ there exists $n \in \mathbb{N}$ such that $D^{n}(x)=0$. The set of locally nilpotent derivations $D: B \rightarrow B$ is denoted $\operatorname{LND}(B)$. One defines

$$
\operatorname{ML}(B)=\bigcap_{D \in \operatorname{LND}(B)} \operatorname{ker} D \quad \text { and } \quad \operatorname{FML}(B)=\bigcap_{D \in \operatorname{LND}(B)} \operatorname{Frac}(\operatorname{ker} D),
$$

where in the second case $B$ is assumed to be a domain and the intersection is taken in Frac $B$. If $\mathbf{k}$ is a field of characteristic zero and $B$ is a $\mathbf{k}$-domain then $\mathbf{k} \subseteq \operatorname{ML}(B) \subseteq \operatorname{FML}(B)$, and if $\operatorname{FML}(B)=\mathbf{k}$ then we say that $B$ has trivial FML-invariant.

Let $\mathbf{k}$ be an algebraically closed field of characteristic zero and $B$ an affine $\mathbf{k}$-domain. It remained an open question for some time whether the condition $\operatorname{ML}(B)=\mathbf{k}$ implied that $B$ is rational over $\mathbf{k}$ (one says that $B$ is rational over $\mathbf{k}$ if the field extension $\operatorname{Frac}(B) / \mathbf{k}$ is purely transcendental). However, Liendo gave examples in Lie10 (and so did Popov in Pop11) showing that the implication is false. Liendo then conjectured that the stronger condition $\operatorname{FML}(B)=\mathbf{k}$ would imply that $B$ is rational or at least unirational over $\mathbf{k}$ (one says that $B$ is unirational over $\mathbf{k}$ if there exists a purely transcendental field extension $F / \mathbf{k}$ of finite transcendence degree such that $\mathbf{k} \subseteq \operatorname{Frac}(B) \subseteq F)$. Then the following result was proved:

Unirationality Theorem. Let $\mathbf{k}$ be an algebraically closed field of characteristic zero and $B$ an affine $\mathbf{k}$-domain satisfying $\operatorname{FML}(B)=\mathbf{k}$. Then $B$ is unirational over $\mathbf{k}$.

This statement follows from either one of [AFK ${ }^{+} 13$, Prop. 5.1] or [Pop14, Thm 4]. Moreover, examples are given in Pop13 showing that $B$, in the above statement, is not necessarily rational over $\mathbf{k}$.

2010 Mathematics Subject Classification. Primary: 14R10. Secondary: 14R20, 14M20, 14R05.

Key words and phrases. Locally nilpotent derivation, unirational variety, affine variety, affine space.

Research supported by a grant from NSERC Canada. 
This article investigates what becomes of the Unirationality Theorem when $\mathbf{k}$ is not assumed to be algebraically closed. It is certainly the case that the condition $\operatorname{FML}(B)=\mathbf{k}$ implies that $B$ is geometrically unirational, i.e., that $\overline{\mathbf{k}} \otimes_{\mathbf{k}} B$ is unirational over $\overline{\mathbf{k}}$, where $\overline{\mathbf{k}}$ denotes the algebraic closure of $\mathbf{k}$ (this follows from the Unirationality Theorem and some straightforward technique, see Cor. 3.2). The aim of this article is to show that $\operatorname{FML}(B)=\mathbf{k}$ implies that $B$ satisfies a condition stronger than geometric unirationality. Before describing this result, let us make a few remarks about Sections 1 and 2 ,

Given a field $\mathbf{k}$ and an affine $\mathbf{k}$-domain $B$, let $\chi_{\mathbf{k}}(B)$ be the set of prime ideals $\mathfrak{p}$ of $B$ such that the $\kappa\left(\mathfrak{p}\right.$ )-algebra $\kappa(\mathfrak{p}) \otimes_{\mathbf{k}} B$ can be embedded in a polynomial ring in finitely many variables over $\kappa(\mathfrak{p})$, where we write $\kappa(\mathfrak{p})=B_{\mathfrak{p}} / \mathfrak{p} B_{\mathfrak{p}}$ for each $\mathfrak{p} \in \operatorname{Spec} B$. It is interesting to consider the class of affine $\mathbf{k}$-domains $B$ satisfying the condition that $X_{\mathbf{k}}(B)$ has nonempty interior (i.e., some nonempty open subset of Spec $B$ is included in $X_{\mathbf{k}}(B)$ ). Ex. 1.15 shows that it is possible for $X_{\mathbf{k}}(B)$ to be dense in $\operatorname{Spec} B$ and to have empty interior, so the condition " $\mathcal{X}_{\mathbf{k}}(B)$ has nonempty interior" is strictly stronger than $X_{\mathbf{k}}(B)$ being dense in $\operatorname{Spec} B$. Although this implies that $X_{\mathbf{k}}(B)$ is not always a constructible subset of Spec $B$, the main result of Section 1 (Thm 1.7) asserts that certain sets closely related to $X_{\mathbf{k}}(B)$ are constructible. Cor. 1.13 asserts that an affine $\mathbf{k}$-domain $B$ satisfies the condition " $\mathcal{X}_{\mathbf{k}}(B)$ has nonempty interior" if and only if the $\operatorname{Frac}(B)$-algebra $\operatorname{Frac}(B) \otimes_{\mathbf{k}} B$ can be embedded in a polynomial ring $(\operatorname{Frac} B)\left[X_{1}, \ldots, X_{n}\right]$ for some $n$.

Section 2 recalls (from Dai18]) some properties of the invariant $\mathscr{K}(B)$ of the $\operatorname{ring} B$. These facts are needed in Section 3 .

The main result of this paper (Thm 3.8) states that if $\mathbf{k}$ is a field of characteristic zero and $B$ is an affine $\mathbf{k}$-domain satisfying $\operatorname{FML}(B)=\mathbf{k}$ then $X_{\mathbf{k}}(B)$ has nonempty interior. Our proof makes use of Thm 1.7 and of some results from Pop14. In the special case where $\mathbf{k}$ is algebraically closed, our result states that if $\operatorname{FML}(B)=\mathbf{k}$ then $B$ can be embedded in a polynomial ring over $\mathbf{k}$, which is stronger than the statement that $B$ is unirational over $\mathbf{k}$.

To the notations and conventions already introduced in the above text, we add the following. We write $\subseteq$ for inclusion, $\subset$ for strict inclusion, and $\backslash$ for set difference. We adopt the convention that $0 \in \mathbb{N}$. If $A$ is a ring and $n \in \mathbb{N}, A^{[n]}$ denotes a polynomial ring in $n$ variables over $A$; if $\mathbf{k}$ is a field, $\mathbf{k}^{(n)}$ denotes the field of fractions of $\mathbf{k}^{[n]}$. We write $\operatorname{trdeg}_{K}(L)$ or $\operatorname{trdeg}(L: K)$ for the transcendence degree of a field extension $L / K$. If $A \subseteq B$ are domains, the transcendence degree of $B$ over $A$ is

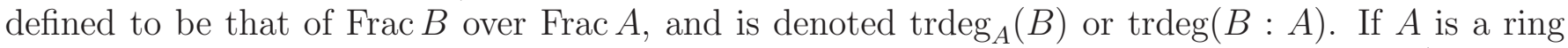
then $A^{*}$ is its group of units, $\operatorname{dim} A$ is the Krull dimension of $A$ and if $a \in A$ then $A_{a}=S^{-1} A$ where $S=\left\{1, a, a^{2}, \ldots\right\}$.

\section{EMBEDDings In POLYNOMIAL ALGEBRAS}

Throughout this section, $\mathbf{k}$ is an arbitrary field.

Given a k-algebra $B$ and $n \in \mathbb{N}$, we write $B \subseteq \mathbf{k}^{[n]}$ as an abbreviation for the sentence: there exists an injective homomorphism of $\mathbf{k}$-algebras from $B$ to a polynomial algebra in $n$ variables over $\mathbf{k}$.

It follows that if $B$ is a $\mathbf{k}$-algebra, $K$ an extension field of $\mathbf{k}$ and $n \in \mathbb{N}$, the notation $K \otimes_{\mathbf{k}} B \subseteq K^{[n]}$ means: there exists an injective homomorphism of $K$-algebras from $K \otimes_{\mathbf{k}} B$ to a polynomial algebra in $n$ variables over $K$. 
1.1. Notation. Given an algebra $B$ over a field $\mathbf{k}$, we write $\operatorname{PE}_{\mathbf{k}}(B)$ for the class of all field extensions $K / \mathbf{k}$ satisfying

$$
K \otimes_{\mathbf{k}} B \subseteq K^{[n]} \text { for some } n \in \mathbb{N} .
$$

The notation "PE" stands for "polynomial embedding" (in the sense of "embedding in a polynomial ring"). Although $\mathrm{PE}_{\mathbf{k}}(B)$ is not necessarily a set 1 there is no harm in using set notations such as " $K / \mathbf{k} \in \mathrm{PE}_{\mathbf{k}}(B) "$ or " $\mathrm{PE}_{\mathbf{k}}(B) \neq \varnothing$ " (whose meanings are obvious). It follows from part (c) of the following fact that if $B$ is finitely generated then $\mathrm{PE}_{\mathbf{k}}(B)$ is the class of extensions $K / \mathbf{k}$ satisfying $K \otimes_{\mathbf{k}} B \subseteq K^{[\operatorname{dim} B]}$.

1.2. Lemma. Let $\mathbf{k}$ be a field, $B$ a finitely generated $\mathbf{k}$-algebra and $K$ an extension field of $\mathbf{k}$.

(a) $K \otimes_{\mathbf{k}} B$ is a finitely generated $K$-algebra and $\operatorname{dim}\left(K \otimes_{\mathbf{k}} B\right)=\operatorname{dim} B$.

(b) If $n \in \mathbb{N}$ is such that $K \otimes_{\mathbf{k}} B \subseteq K^{[n]}$, then $n \geq \operatorname{dim} B$.

(c) If there exists $n \in \mathbb{N}$ such that $K \otimes_{\mathbf{k}} B \subseteq K^{[n]}$, then $K \otimes_{\mathbf{k}} B \subseteq K^{[\operatorname{dim} B]}$.

Proof. It is clear that $K \otimes_{\mathbf{k}} B$ is finitely generated. Let $d=\operatorname{dim} B$. By Noether's Normalization Lemma there exists an injective $\mathbf{k}$-homomorphism $\mathbf{k}^{[d]} \rightarrow B$ which is also integral. Applying the functor $K \otimes_{\mathbf{k}}\left({ }_{-}\right)$gives an injective and integral $K$-homomorphism from $K \otimes_{\mathbf{k}} \mathbf{k}^{[d]}=K^{[d]}$ to $K \otimes_{\mathbf{k}} B$, so $\operatorname{dim}\left(K \otimes_{\mathbf{k}} B\right)=d$. This proves (a). Assertions (b) and (c) follow from Lemma B of [Eak72].

1.3. Lemma. Let $B$ be an algebra over a field $\mathbf{k}$ and suppose that $K / \mathbf{k} \in \mathrm{PE}_{\mathbf{k}}(B)$.

(a) Every overfield $L$ of $K$ satisfies $L / \mathbf{k} \in \mathrm{PE}_{\mathbf{k}}(B)$.

(b) If $B$ is finitely generated as a $\mathbf{k}$-algebra then there exists a finitely generated field extension $K_{0} / \mathbf{k}$ such that $\mathbf{k} \subseteq K_{0} \subseteq K$ and $K_{0} / \mathbf{k} \in \mathrm{PE}_{\mathbf{k}}(B)$.

Proof. (a) For some $n \in \mathbb{N}$, there exists an injective $K$-homomorphism $K \otimes_{\mathbf{k}} B \rightarrow K^{[n]}$. Applying $L \otimes_{K}($ _ $)$ gives an injective $L$-homomorphism from $L \otimes_{K}\left(K \otimes_{\mathbf{k}} B\right)=L \otimes_{\mathbf{k}} B$ to $L \otimes_{K} K^{[n]}=L^{[n]}$.

(b) For some $n \in \mathbb{N}$, there exists an injective $K$-homomorphism $\varphi: K \otimes_{\mathbf{k}} B \rightarrow K\left[X_{1}, \ldots, X_{n}\right]=$ $K^{[n]}$. Choose $b_{1}, \ldots, b_{s} \in B$ such that $B=\mathbf{k}\left[b_{1}, \ldots, b_{s}\right]$. There exists a finite subset $S$ of $K$ that contains all coefficients of the polynomials $\varphi\left(1 \otimes b_{i}\right) \in K\left[X_{1}, \ldots, X_{n}\right], 1 \leq i \leq s$. Define $K_{0}=\mathbf{k}(S)$, then the image of the composite $K_{0} \otimes_{\mathbf{k}} B \rightarrow K \otimes_{\mathbf{k}} B \stackrel{\varphi}{\rightarrow} K\left[X_{1}, \ldots, X_{n}\right]$ is included in $K_{0}\left[X_{1}, \ldots, X_{n}\right]$, so $K_{0} \otimes_{\mathbf{k}} B \subseteq K_{0}^{[n]}$ and hence $K_{0} / \mathbf{k} \in \mathrm{PE}_{\mathbf{k}}(B)$.

1.4. Lemma. Let $B$ be an algebra over a field $\mathbf{k}$. If $\mathrm{PE}_{\mathbf{k}}(B) \neq \varnothing$ then $B$ is geometrically integral, i.e., $K \otimes_{\mathbf{k}} B$ is a domain for every extension field $K$ of $\mathbf{k}$.

Proof. Let $K$ be an extension field of $\mathbf{k}$. Choose an element $L / \mathbf{k}$ of $\mathrm{PE}_{\mathbf{k}}(B)$, and choose an algebraically closed field $M$ satisfying $L \subseteq M$ and $\operatorname{trdeg}_{\mathbf{k}}(M) \geq \operatorname{trdeg}_{\mathbf{k}}(K)$. Then there exists a $\mathbf{k}$-homomorphism $K \rightarrow M$, so the fact that $B$ is a flat $\mathbf{k}$-module implies that $K \otimes_{\mathbf{k}} B$ is a subring of $M \otimes_{\mathbf{k}} B$. We have $M / \mathbf{k} \in \mathrm{PE}_{\mathbf{k}}(B)$ by Lemma 1.3, so $M \otimes_{\mathbf{k}} B \subseteq M^{[n]}$ for some $n$, so $M \otimes_{\mathbf{k}} B$ is a domain and hence $K \otimes_{\mathbf{k}} B$ is a domain.

\footnotetext{
${ }^{1}$ For instance if $B \subseteq \mathbf{k}^{[n]}$ then $\mathrm{PE}_{\mathbf{k}}(B)$ is the class of all field extensions $K / \mathbf{k}$, which is not a set in the sense of the ZFC axiomatization.
} 
1.5. Lemma. Consider a tensor product of rings

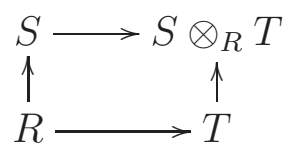

where all homomorphisms are injective.

(a) Suppose that $S$ is a free $R$-module and that there exists a basis $\mathcal{E}$ of $S$ over $R$ such that $1 \in \mathcal{E}$. Then $S \cap T=R$.

(b) If $R, S, T$ and $S \otimes_{R} T$ are domains, and if $\left(s_{j}\right)_{j \in J}$ is a family of elements of $S$ which is a transcendence basis of Frac $S$ over Frac $R$, then $\left(s_{j} \otimes 1\right)_{j \in J}$ is a transcendence basis of $\operatorname{Frac}\left(S \otimes_{R} T\right)$ over Frac $T$. In particular, $\operatorname{trdeg}_{T}\left(S \otimes_{R} T\right)=\operatorname{trdeg}_{R} S$.

Proof. Exercise left to the reader.

1.6. Notations. Let $\mathbf{k}$ be a field, let $R$ and $B$ be k-algebras, let $N \in \mathbb{N}$ and let $R[X]=R\left[X_{1}, \ldots, X_{N}\right]=$ $R^{[N]}$. Let $\Psi: B \rightarrow R[X]$ be a $\mathbf{k}$-homomorphism. Then for each prime ideal $\mathfrak{p} \in \operatorname{Spec} R$ we define the following notations:

- $\varphi_{\mathfrak{p}}: R \rightarrow \kappa(\mathfrak{p})$ is the canonical homomorphism, where $\kappa(\mathfrak{p})=R_{\mathfrak{p}} / \mathfrak{p} R_{\mathfrak{p}}$.

- $\tilde{\varphi}_{\mathfrak{p}}: R[X] \rightarrow \kappa(\mathfrak{p})[X]$ is the induced homomorphism satisfying $\tilde{\varphi}_{\mathfrak{p}}\left(X_{i}\right)=X_{i}$ for all $i$.

- $\Psi^{\mathfrak{p}}: B \rightarrow \kappa(\mathfrak{p})[X]$ is the composition $B \stackrel{\Psi}{\rightarrow} R[X] \stackrel{\tilde{\varphi}_{\mathfrak{p}}}{\longrightarrow} \kappa(\mathfrak{p})[X]$.

- $\hat{\Psi}^{\mathfrak{p}}: \kappa(\mathfrak{p}) \otimes_{\mathbf{k}} B \rightarrow \kappa(\mathfrak{p})[X]$ is given by the universal property of the pushout:

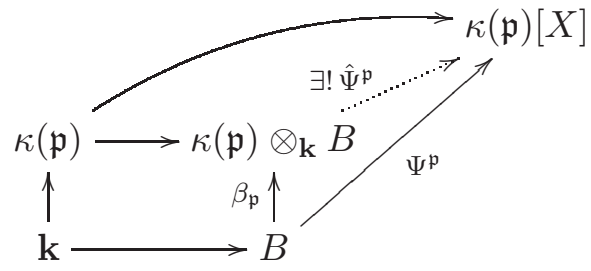

Note that $\Psi^{\mathfrak{p}}$ is a $\mathbf{k}$-homomorphism and that $\hat{\Psi}^{\mathfrak{p}}$ is a $\kappa(\mathfrak{p})$-homomorphism. We define:

$$
X_{\mathbf{k}}(\Psi)=\left\{\mathfrak{p} \in \operatorname{Spec} R \mid \hat{\Psi}^{\mathfrak{p}} \text { is injective }\right\} .
$$

1.7. Theorem. Let the setup be as in paragraph 1.6. If $B$ and $R$ are affine $\mathbf{k}$-domains then the following hold.

(a) $X_{\mathbf{k}}(\Psi)$ is a constructible subset of $\operatorname{Spec} R$.

(b) If $B$ is geometrically integral and $\mathfrak{m}$ is a maximal ideal of $R$, then $\mathfrak{m} \in X_{\mathbf{k}}(\Psi)$ if and only if $\Psi^{\mathfrak{m}}$ is injective.

Proof. Let $n=\operatorname{dim} B$. We first prove (B) . Let $\mathfrak{m}$ be a maximal ideal of $R$ and consider diagram (1) with $\mathfrak{p}=\mathfrak{m}$. Since the canonical homomorphism $B \rightarrow \kappa(\mathfrak{m}) \otimes_{\mathbf{k}} B$ is injective, it is clear that if $\mathfrak{m} \in X_{\mathbf{k}}(\Psi)$ then $\Psi^{\mathfrak{m}}$ is injective. Conversely, suppose that $\Psi^{\mathfrak{m}}$ is injective. Then $\operatorname{trdeg}_{\mathbf{k}}\left(\operatorname{im} \Psi^{\mathfrak{m}}\right)=n$. By (11), we have $\operatorname{im}\left(\Psi^{\mathfrak{m}}\right) \subseteq \operatorname{im}\left(\hat{\Psi}^{\mathfrak{m}}\right)$, so $\operatorname{trdeg}_{\mathbf{k}}\left(\operatorname{im} \hat{\Psi}^{\mathfrak{m}}\right) \geq n$. Since $\kappa(\mathfrak{m})$ is an algebraic extension of $\mathbf{k}, \operatorname{trdeg}_{\kappa(\mathfrak{m})}\left(\operatorname{im} \hat{\Psi}^{\mathfrak{m}}\right)=\operatorname{trdeg}_{\mathbf{k}}\left(\operatorname{im} \hat{\Psi}^{\mathfrak{m}}\right) \geq n$. As $B$ is geometrically integral, $\operatorname{dom}\left(\hat{\Psi}^{\mathfrak{m}}\right)=\kappa(\mathfrak{m}) \otimes_{\mathbf{k}}$ $B$ is an affine $\kappa(\mathfrak{m})$-domain of dimension $n$ (by Lemma 1.2); it follows that $\operatorname{trdeg}(\mathfrak{m})\left(\operatorname{im} \hat{\Psi}^{\mathfrak{m}}\right) \geq$ $\operatorname{trdeg}_{\kappa(\mathfrak{m})}\left(\operatorname{dom} \hat{\Psi}^{\mathfrak{m}}\right)$, so $\hat{\Psi}^{\mathfrak{m}}$ is injective and $\mathfrak{m} \in X_{\mathbf{k}}(\Psi)$. So (b) is proved. 
To prove (国), we may assume that $X_{\mathbf{k}}(\Psi) \neq \varnothing$. Then $\kappa(\mathfrak{p}) \otimes_{\mathbf{k}} B \subseteq \kappa(\mathfrak{p})[X]=\kappa(\mathfrak{p})^{[N]}$ for any $\mathfrak{p} \in \mathcal{X}_{\mathbf{k}}(\Psi)$, so $B$ is geometrically integral by Lemma 1.4, also, Lemma $1.2 \operatorname{implies}$ that $N \geq \operatorname{dim} B=n$ and:

$$
\text { for each } \mathfrak{p} \in \operatorname{Spec} R, \kappa(\mathfrak{p}) \otimes_{\mathbf{k}} B \text { is an affine } \kappa(\mathfrak{p}) \text {-domain of dimension } n \text {. }
$$

The first step in the proof of (国) consists in proving:

$$
\text { If } N=\operatorname{dim} B \text { and } 0 \in \mathcal{X}_{\mathbf{k}}(\Psi) \text { then } \mathcal{X}_{\mathbf{k}}(\Psi) \text { contains a nonempty open subset of Spec } R \text {. }
$$

Here, 0 stands for the zero ideal of $R$. Note that the codomain of $\Psi$ is $R[X]=R\left[X_{1}, \ldots, X_{n}\right]=R^{[n]}$ (because $N=n$ ) and consider the homomorphism $\hat{\Psi}: R \otimes_{\mathbf{k}} B \rightarrow R[X]$ given by $\hat{\Psi}(r \otimes b)=r \Psi(b)$. Let $K=\operatorname{Frac} R$. Since $0 \in X_{\mathbf{k}}(\Psi), \hat{\Psi}^{0}: K \otimes_{\mathbf{k}} B \rightarrow K[X]$ is an injective $K$-homomorphism. For each $\mathfrak{p} \in \operatorname{Spec} R$, there is a commutative diagram

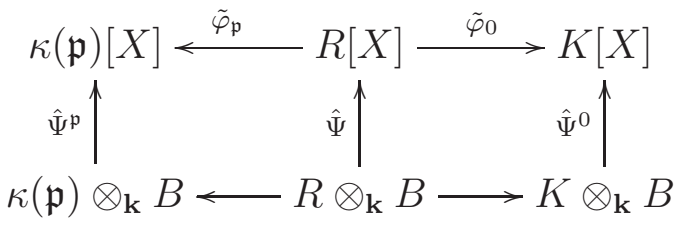

Let $j \in\{1, \ldots, n\}$. Since $K \otimes_{\mathbf{k}} B$ is an affine $K$-domain of dimension $n$ and $\hat{\Psi}^{0}$ is an injective $K$ homomorphism, $X_{j}$ is algebraic over $\hat{\Psi}^{0}\left(K \otimes_{\mathbf{k}} B\right)$; since $\hat{\Psi}^{0}\left(K \otimes_{\mathbf{k}} B\right)$ is a localization of $\hat{\Psi}\left(R \otimes_{\mathbf{k}} B\right)$, it follows that $X_{j}$ is algebraic over $\hat{\Psi}\left(R \otimes_{\mathrm{k}} B\right)$, so there exists a nonzero polynomial in one variable $P_{j}(T) \in \hat{\Psi}\left(R \otimes_{\mathbf{k}} B\right)[T]$ such that $P_{j}\left(X_{j}\right)=0$. Let $c_{j} \in R \otimes_{\mathbf{k}} B$ be such that $\hat{\Psi}\left(c_{j}\right) \in \hat{\Psi}\left(R \otimes_{\mathbf{k}} B\right) \backslash\{0\}$ is the leading coefficient of $P_{j}(T)$. Since $\hat{\Psi}\left(c_{j}\right)$ is a nonzero polynomial in $R[X]$, the ideal $I_{j}$ of $R$ generated by the coefficients of $\hat{\Psi}\left(c_{j}\right)$ is nonzero, and consequently $U_{j}=\operatorname{Spec} R \backslash V\left(I_{j}\right)$ is a nonempty open subset of Spec $R$. If $\mathfrak{p} \in U_{j}$ then $\hat{\Psi}\left(c_{j}\right) \notin \mathfrak{p}[X]$ and consequently $\tilde{\varphi}_{\mathfrak{p}}\left(\hat{\Psi}\left(c_{j}\right)\right) \neq 0$. Since $\tilde{\varphi}_{\mathfrak{p}}\left(\hat{\Psi}\left(c_{j}\right)\right)$ is a coefficient of the polynomial $P_{j}^{\left(\tilde{\varphi}_{\mathfrak{p}}\right)}(T) \in \hat{\Psi}^{\mathfrak{p}}\left(\kappa(\mathfrak{p}) \otimes_{\mathbf{k}} B\right)[T]$, we have $P_{j}^{\left(\tilde{\varphi}_{\mathfrak{p}}\right)}(T) \neq 0$. Now $\kappa(\mathfrak{p}) \subseteq \operatorname{im}\left(\hat{\Psi}^{\mathfrak{p}}\right) \subseteq \kappa(\mathfrak{p})[X], P_{j}^{\left(\tilde{\varphi}_{\mathfrak{p}}\right)}(T)$ is a nonzero polynomial in $T$ with coefficients in the $\operatorname{ring} \operatorname{im}\left(\hat{\Psi}^{\mathfrak{p}}\right)$, and $P_{j}^{\left(\tilde{\varphi}_{\mathfrak{p}}\right)}\left(X_{j}\right)=\tilde{\varphi}_{\mathfrak{p}}\left(P_{j}\left(X_{j}\right)\right)=0$; so $X_{j}$ is algebraic over $\operatorname{im}\left(\hat{\Psi}^{\mathfrak{p}}\right)$.

Consider the nonempty open subset $U=\bigcap_{j=1}^{n} U_{j}$ of Spec $R$. Let $\mathfrak{p} \in U$. The preceding paragraph implies that $X_{1}, \ldots, X_{n}$ are algebraic over $\operatorname{im}\left(\hat{\Psi}^{\mathfrak{p}}\right)$, so $\kappa(\mathfrak{p})[X]$ is algebraic over $\operatorname{im}\left(\hat{\Psi}^{\mathfrak{p}}\right)$ and consequently $\operatorname{trdeg}_{\kappa(\mathfrak{p})}\left(\operatorname{im} \hat{\Psi}^{\mathfrak{p}}\right)=n$; as $\operatorname{dom}\left(\hat{\Psi}^{\mathfrak{p}}\right)=\kappa(\mathfrak{p}) \otimes_{\mathbf{k}} B$ is an affine $\kappa(\mathfrak{p})$-domain of dimension $n$ and $\hat{\Psi}^{\mathfrak{p}}$ is a $\kappa(\mathfrak{p})$-homomorphism, it follows that $\hat{\Psi}^{\mathfrak{p}}$ is injective and hence that $\mathfrak{p} \in \mathcal{X}_{\mathbf{k}}(\Psi)$. This shows that $U \subseteq X_{\mathbf{k}}(\Psi)$, so (2) is proved. Next, we generalize (21) slightly. Let us prove:

$$
\text { If } 0 \in X_{\mathbf{k}}(\Psi) \text { then } X_{\mathbf{k}}(\Psi) \text { contains a nonempty open subset of } \operatorname{Spec} R \text {. }
$$

By assumption, $\hat{\Psi}^{0}: \kappa(0) \otimes_{\mathbf{k}} B \rightarrow \kappa(0)[X]$ is injective. By Lemma B of [Eak72] applied to $\kappa(0) \subseteq$ $\kappa(0) \otimes_{\mathbf{k}} B \subseteq \kappa(0)[X]$, there exists a $\kappa(0)$-homomorphism $\rho: \kappa(0)\left[X_{1}, \ldots, X_{N}\right] \rightarrow \kappa(0)\left[Y_{1}, \ldots, Y_{n}\right]=$ $\kappa(0)^{[n]}$ such that $\rho \circ \hat{\Psi}^{0}: \kappa(0) \otimes_{\mathbf{k}} B \rightarrow \kappa(0)[Y]$ is injective. Since Frac $R=\kappa(0)$, there exists $r \in R \backslash\{0\}$ such that if we define $A=R[1 / r]$ then the image of the composite $B \rightarrow \kappa(0) \otimes_{\mathbf{k}} B \stackrel{\rho \circ \hat{\Psi}^{0}}{\longrightarrow} \kappa(0)[Y]$ is included in $A[Y]$. So there is a k-homomorphism $\Psi_{1}: B \rightarrow A[Y]$ such that the following diagram 
commutes:

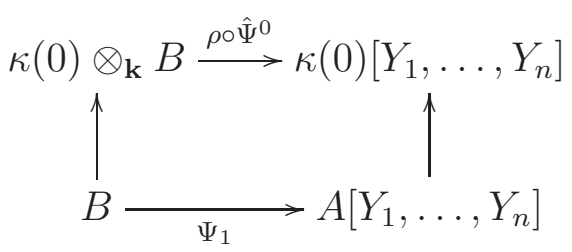

Since $\hat{\Psi}_{1}^{0}$ coincides exactly with $\rho \circ \hat{\Psi}^{0}$, which is injective, we have $0 \in \mathcal{X}_{\mathbf{k}}\left(\Psi_{1}\right)$, so $\Psi_{1}$ satisfies the hypothesis of (2). Thus $X_{\mathbf{k}}\left(\Psi_{1}\right)$ contains a nonempty open subset of Spec $A$. It is straightforward to check that the open immersion $\operatorname{Spec} A \rightarrow \operatorname{Spec} R$ maps $X_{\mathbf{k}}\left(\Psi_{1}\right)$ into $X_{\mathbf{k}}(\Psi)$, so $X_{\mathbf{k}}(\Psi)$ contains a nonempty open subset of Spec $R$ and (3) is proved. From this, let us deduce that the following is true:

If $X_{\mathbf{k}}(\Psi)$ is dense in $\operatorname{Spec} R$, then $X_{\mathbf{k}}(\Psi)$ contains a nonempty open subset of Spec $R$.

So assume that $X_{\mathbf{k}}(\Psi)$ is dense in Spec $R$. Let $\left(b_{1}, \ldots, b_{n}\right)$ be a family of elements of $B$ that is a transcendence basis of Frac $B$ over $\mathbf{k}$ (recall that $n=\operatorname{dim} B=\operatorname{trdeg}_{\mathbf{k}} B$ ), and consider the family $\left(\Psi\left(b_{i}\right)\right)_{i=1}^{n}$ in $R[X]$; we claim that $\left(\Psi\left(b_{i}\right)\right)_{i=1}^{n}$ is algebraically independent over $R$. Indeed, consider $G \in R\left[T_{1}, \ldots, T_{n}\right]=R^{[n]}$ such that $G\left(\Psi\left(b_{1}\right), \ldots, \Psi\left(b_{n}\right)\right)=0$. Let $\mathfrak{p} \in X_{\mathbf{k}}(\Psi)$. Then the polynomial $G^{\left(\varphi_{\mathfrak{p}}\right)} \in \kappa(\mathfrak{p})\left[T_{1}, \ldots, T_{n}\right]$ satisfies $G^{\left(\varphi_{\mathfrak{p}}\right)}\left(\hat{\Psi}^{\mathfrak{p}}\left(1 \otimes b_{1}\right), \ldots, \hat{\Psi}^{\mathfrak{p}}\left(1 \otimes b_{n}\right)\right)=G^{\left(\varphi_{\mathfrak{p}}\right)}\left(\Psi^{\mathfrak{p}}\left(b_{1}\right), \ldots, \Psi^{\mathfrak{p}}\left(b_{n}\right)\right)=$ $G^{\left(\varphi_{\mathfrak{p}}\right)}\left(\tilde{\varphi}_{\mathfrak{p}}\left(\Psi\left(b_{1}\right)\right), \ldots, \tilde{\varphi}_{\mathfrak{p}}\left(\Psi\left(b_{1}\right)\right)\right)=\tilde{\varphi}_{\mathfrak{p}}\left(G\left(\Psi\left(b_{1}\right), \ldots, \Psi\left(b_{n}\right)\right)\right)=\tilde{\varphi}_{\mathfrak{p}}(0)=0$. Since the family $\left(1 \otimes b_{i}\right)_{i=1}^{n}$ of elements of $\kappa(\mathfrak{p}) \otimes_{\mathbf{k}} B$ is algebraically independent over $\kappa(\mathfrak{p})$ (Lemma 1.5), and since $\hat{\Psi}^{\mathfrak{p}}$ is an injective $\kappa(\mathfrak{p})$-homomorphism, the family $\left(\hat{\Psi}^{\mathfrak{p}}\left(1 \otimes b_{i}\right)\right)_{i=1}^{n}$ of elements of $\kappa(\mathfrak{p})[X]$ is algebraically independent over $\kappa(\mathfrak{p})$; so $G^{\left(\varphi_{\mathfrak{p}}\right)}=0$ and hence $G \in \mathfrak{p}\left[T_{1}, \ldots, T_{n}\right]$. Since this is true for every $\mathfrak{p} \in X_{\mathbf{k}}(\Psi)$, and since $\mathcal{X}_{\mathbf{k}}(\Psi)$ is dense in Spec $R$, it follows that $G=0$. This proves that $\left(\Psi\left(b_{i}\right)\right)_{i=1}^{n}$ is algebraically independent over $R$. Since $\varphi_{0}: R \rightarrow \kappa(0)$ is the inclusion of $R$ in its field of fractions, the image $\left(\tilde{\varphi}_{0}\left(\Psi\left(b_{i}\right)\right)\right)_{i=1}^{n}$ of $\left(\Psi\left(b_{i}\right)\right)_{i=1}^{n}$ by $\tilde{\varphi}_{0}: R[X] \rightarrow \kappa(0)[X]$ is a family of elements of $\kappa(0)[X]$ that is algebraically independent over $\kappa(0)$. Since $\tilde{\varphi}_{0}\left(\Psi\left(b_{i}\right)\right)=\Psi^{0}\left(b_{i}\right)=\hat{\Psi}^{0}\left(1 \otimes b_{i}\right)$, $\left(\tilde{\varphi}_{0}\left(\Psi\left(b_{i}\right)\right)\right)_{i=1}^{n}$ is a family of elements of $\operatorname{im} \hat{\Psi}^{0}$, so $\operatorname{trdeg}_{\kappa(0)}\left(\operatorname{im} \hat{\Psi}^{0}\right) \geq n$. By Lemma 1.2 and the fact that $B$ is geometrically integral, dom $\hat{\Psi}^{0}=\kappa(0) \otimes_{\mathbf{k}} B$ is an affine $\kappa(0)$-domain of dimension equal to $\operatorname{dim} B=n, \operatorname{so}_{\operatorname{trdeg}}\left(\lim \hat{\Psi}^{0}\right) \geq \operatorname{trdeg}_{\kappa(0)}\left(\operatorname{dom} \hat{\Psi}^{0}\right)$ and hence $\hat{\Psi}^{0}: \kappa(0) \otimes_{\mathbf{k}} B \rightarrow \kappa(0)[X]$ is injective. Thus $0 \in X_{\mathbf{k}}(\Psi)$, so (3) implies that $X_{\mathbf{k}}(\Psi)$ contains a nonempty open subset of Spec $R$. So (4) is proved.

For each $\mathfrak{p} \in \operatorname{Spec} R$, we write $V(\mathfrak{p})=\{\mathfrak{q} \in \operatorname{Spec} R \mid \mathfrak{p} \subseteq \mathfrak{q}\}$. To show that $X_{\mathbf{k}}(\Psi)$ is constructible, it suffices (by Prop. 6.C of [Mat80]) to prove the following:

$$
\begin{aligned}
& \text { If } \mathfrak{p} \in \operatorname{Spec} R \text { is such that } V(\mathfrak{p}) \cap \mathcal{X}_{\mathbf{k}}(\Psi) \text { is dense in } V(\mathfrak{p}) \text {, then } V(\mathfrak{p}) \cap \mathcal{X}_{\mathbf{k}}(\Psi) \text { contains a } \\
& \text { nonempty open subset of } V(\mathfrak{p}) \text {. }
\end{aligned}
$$

To prove this, consider $\mathfrak{p} \in \operatorname{Spec} R$ such that $V(\mathfrak{p}) \cap X_{\mathbf{k}}(\Psi)$ is dense in $V(\mathfrak{p})$. The canonical homomorphism $R \rightarrow R / \mathfrak{p}$ extends to a homomorphism $R[X] \rightarrow(R / \mathfrak{p})[X]$ that sends each $X_{i}$ to itself; let us define $\Psi_{1}: B \rightarrow(R / \mathfrak{p})[X]$ to be the composition $B \stackrel{\Psi}{\rightarrow} R[X] \rightarrow(R / \mathfrak{p})[X]$. Then it is straightforward to verify that the canonical homeomorphism $f: \operatorname{Spec}(R / \mathfrak{p}) \rightarrow V(\mathfrak{p})$ satisfies $f\left(X_{\mathbf{k}}\left(\Psi_{1}\right)\right)=V(\mathfrak{p}) \cap X_{\mathbf{k}}(\Psi)$. Since $V(\mathfrak{p}) \cap X_{\mathbf{k}}(\Psi)$ is dense in $V(\mathfrak{p})$, it follows that $X_{\mathbf{k}}\left(\Psi_{1}\right)$ is dense in $\operatorname{Spec}(R / \mathfrak{p})$. By (4), we obtain that $X_{\mathbf{k}}\left(\Psi_{1}\right)$ contains a nonempty open subset of $\operatorname{Spec}(R / \mathfrak{p})$. So 
$f\left(X_{\mathbf{k}}\left(\Psi_{1}\right)\right)=V(\mathfrak{p}) \cap X_{\mathbf{k}}(\Psi)$ contains a nonempty open subset of $V(\mathfrak{p})$. So (5) is proved, and so is the Theorem.

1.8. Corollary. Let $\mathbf{k}$ be a field and $B$ an affine $\mathbf{k}$-domain such that $\mathrm{PE}_{\mathbf{k}}(B) \neq \varnothing$.

(a) There exists an affine $\mathbf{k}$-domain $R$ satisfying $\operatorname{Frac}(R) / \mathbf{k} \in \mathrm{PE}_{\mathbf{k}}(B)$.

(b) For any $R$ as in (国), there exists a nonempty open subset $U$ of $\operatorname{Spec} R$ satisfying

$$
\kappa(\mathfrak{p}) / \mathbf{k} \in \mathrm{PE}_{\mathbf{k}}(B) \text { for all } \mathfrak{p} \in U,
$$

where we write $\kappa(\mathfrak{p})=R_{\mathfrak{p}} / \mathfrak{p} R_{\mathfrak{p}}$.

Proof. Assertion (国) follows from Lemma 1.3. We prove (b). Let $K=\operatorname{Frac} R$. Since $K / \mathbf{k} \in \mathrm{PE}_{\mathbf{k}}(B)$, there exists an injective $K$-homomorphism $\varphi: K \otimes_{\mathbf{k}} B \rightarrow K[X]=K\left[X_{1}, \ldots, X_{n}\right]=K^{[n]}$ (for some $n \in \mathbb{N}$ ). There exists $r \in R \backslash\{0\}$ such that, if we define $A=R[1 / r]$, the image of the composite $B \rightarrow K \otimes_{\mathbf{k}} B \stackrel{\varphi}{\rightarrow} K[X]$ is included in $A[X]$. So we may consider the unique $\mathbf{k}$-homomorphism $\Psi: B \rightarrow A[X]$ that makes

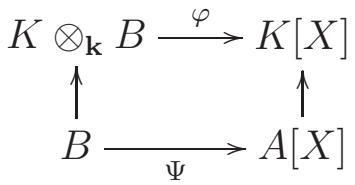

commute. If 0 denotes the zero ideal of $A$ then $\hat{\Psi}^{0}=\varphi$, so $\hat{\Psi}^{0}$ is injective and hence $0 \in X_{\mathbf{k}}(\Psi)$; so $X_{\mathbf{k}}(\Psi)$ is a dense subset of Spec $A$. Since $X_{\mathbf{k}}(\Psi)$ is constructible by Thm 1.7, $X_{\mathbf{k}}(\Psi)$ contains a nonempty open subset $U^{\prime}$ of $\operatorname{Spec} A$. Noting that $f: \operatorname{Spec} A \rightarrow \operatorname{Spec} R$ is an open immersion, we see that $U=f\left(U^{\prime}\right)$ is a nonempty open subset of $\operatorname{Spec} R$ that has the desired property.

1.9. Corollary. Let $\mathbf{k}$ be a field and $B$ an affine $\mathbf{k}$-domain such that $\mathrm{PE}_{\mathbf{k}}(B) \neq \varnothing$.

(a) There exists a finite extension $\mathbf{k}^{\prime}$ of $\mathbf{k}$ satisfying $\mathbf{k}^{\prime} / \mathbf{k} \in \mathrm{PE}_{\mathbf{k}}(B)$.

(b) The algebraic closure $\overline{\mathbf{k}}$ of $\mathbf{k}$ satisfies $\overline{\mathbf{k}} / \mathbf{k} \in \mathrm{PE}_{\mathbf{k}}(B)$.

Proof. By Cor. 1.8, there exists an affine $\mathbf{k}$-domain $R$ such that $\operatorname{Frac}(R) / \mathbf{k} \in \operatorname{PE}_{\mathbf{k}}(B)$ and a nonempty open subset $U$ of Spec $R$ such that $\kappa(\mathfrak{p}) / \mathbf{k} \in \mathrm{PE}_{\mathbf{k}}(B)$ for all $\mathfrak{p} \in U$, where $\kappa(\mathfrak{p})=R_{\mathfrak{p}} / \mathfrak{p} R_{\mathfrak{p}}$. Choose a maximal ideal $\mathfrak{m}$ of $R$ such that $\mathfrak{m} \in U$. Then $\kappa(\mathfrak{m}) / \mathbf{k} \in \mathrm{PE}_{\mathbf{k}}(B)$ and $\kappa(\mathfrak{m}) / \mathbf{k}$ is a finite extension, so (a) is proved. As $\overline{\mathbf{k}}$ is an overfield of any finite extension $\mathbf{k}^{\prime}$ of $\mathbf{k}$, assertion (b) follows from (a) and Lemma 1.3 .

1.10. Definition. We say that a field extension $L / K$ has the density property if it is finitely generated and the following equivalent conditions hold:

- for some affine $K$-domain $R$ satisfying Frac $R=L, K$-rational points are dense in Spec $R$;

- for every affine $K$-domain $R$ satisfying $\operatorname{Frac} R=L, K$-rational points are dense in $\operatorname{Spec} R$.

Note the following descent property for $\operatorname{PE}_{\mathbf{k}}(B)$ :

1.11. Corollary. Let $\mathbf{k} \subseteq K \subseteq L$ be fields and let $B$ be an affine $\mathbf{k}$-domain. If $L / \mathbf{k} \in \mathrm{PE}_{\mathbf{k}}(B)$ and $L / K$ has the density property, then $K / \mathbf{k} \in \mathrm{PE}_{\mathbf{k}}(B)$. 
Proof. The fact that $L / \mathbf{k} \in \mathrm{PE}_{\mathbf{k}}(B)$ implies that (for some $n$ ) there is an injective $L$-homomorphism from $L \otimes_{\mathbf{k}} B=L \otimes_{K}\left(K \otimes_{\mathbf{k}} B\right)$ to $L^{[n]}$, so $L \otimes_{K}\left(K \otimes_{\mathbf{k}} B\right) \subseteq L^{[n]}$ and consequently $L / K \in \operatorname{PE}_{K}\left(K \otimes_{\mathbf{k}} B\right)$. Since $L / K$ has the density property, it is finitely generated; so there exists an affine $K$-domain $R$ satisfying $\operatorname{Frac} R=L$. Then $\operatorname{Frac}(R) / K \in \mathrm{PE}_{K}\left(K \otimes_{\mathbf{k}} B\right)$, so Cor. 1.8 implies that there exists a nonempty open subset $U$ of $\operatorname{Spec} R$ such that $\kappa(\mathfrak{p}) / K \in \mathrm{PE}_{K}\left(K \otimes_{\mathbf{k}} B\right)$ for all $\mathfrak{p} \in U$, where $\kappa(\mathfrak{p})=R_{\mathfrak{p}} / \mathfrak{p} R_{\mathfrak{p}}$. Since $L / K$ has the density property, there exists a $K$-rational point in $U$, i.e., there exists $\mathfrak{p} \in U$ such that $\kappa(\mathfrak{p})=K$. Thus $K / K \in \mathrm{PE}_{K}\left(K \otimes_{\mathbf{k}} B\right)$ and consequently $K \otimes_{\mathbf{k}} B \subseteq K^{[n]}$. This means that $K / \mathbf{k} \in \mathrm{PE}_{\mathbf{k}}(B)$, so we are done.

1.12. Notation. Given an algebra $B$ over a field $\mathbf{k}$, define

$$
X_{\mathbf{k}}(B)=\left\{\mathfrak{p} \in \operatorname{Spec} B \mid \kappa(\mathfrak{p}) / \mathbf{k} \in \operatorname{PE}_{\mathbf{k}}(B)\right\}=\left\{\mathfrak{p} \in \operatorname{Spec} B \mid \kappa(\mathfrak{p}) \otimes_{\mathbf{k}} B \subseteq \kappa(\mathfrak{p})^{[n]} \text { for some } n \in \mathbb{N}\right\}
$$

where $\kappa(\mathfrak{p})=B_{\mathfrak{p}} / \mathfrak{p} B_{\mathfrak{p}}$. Note that $X_{\mathbf{k}}(B)=\left\{\mathfrak{p} \in \operatorname{Spec} B \mid \kappa(\mathfrak{p}) \otimes_{\mathbf{k}} B \subseteq \kappa(\mathfrak{p})^{[\operatorname{dim} B]}\right\}$ when $B$ is finitely generated, by Lemma 1.2 .

One says that $X_{\mathbf{k}}(B)$ has nonempty interior if some nonempty open subset of $\operatorname{Spec} B$ is included in $X_{\mathbf{k}}(B)$. For reasons explained in the Introduction (and further explained in Section 3), we are interested in $\mathbf{k}$-algebras $B$ such that $X_{\mathbf{k}}(B)$ has nonempty interior.

1.13. Corollary. Given a field $\mathbf{k}$ and an affine $\mathbf{k - d o m a i n ~} B$,

$$
X_{\mathbf{k}}(B) \text { has nonempty interior } \Longleftrightarrow \operatorname{Frac}(B) / \mathbf{k} \in \mathrm{PE}_{\mathbf{k}}(B) \text {. }
$$

Proof. If $X_{\mathbf{k}}(B)$ has nonempty interior then the generic point of Spec $B$ is an element of $X_{\mathbf{k}}(B)$, so $\operatorname{Frac}(B) / \mathbf{k} \in \mathrm{PE}_{\mathbf{k}}(B)$. The converse is the case $R=B$ of Cor. 1.8,

1.14. Remark. Let $\mathbf{k}$ be a field and $B$ an affine $\mathbf{k}$-domain such that $\chi_{\mathbf{k}}(B)$ has nonempty interior. Then the following are equivalent:

(a) $B \subseteq \mathbf{k}^{[\operatorname{dim} B]}$

(b) $\mathbf{k}$-rational points are dense in $\operatorname{Spec} B$

(c) the extension $\operatorname{Frac}(B) / \mathbf{k}$ has the density property

(d) B is unirational over $\mathbf{k}$.

Proof. Let $n=\operatorname{dim} B$. By assumption, there exists a dense open subset $U$ of Spec $B$ such that $U \subseteq X_{\mathbf{k}}(B)$. Then $\kappa(\mathfrak{p}) \otimes_{\mathbf{k}} B \subseteq \kappa(\mathfrak{p})^{[n]}$ for all $\mathfrak{p} \in U$. If (b) holds, there exists a k-rational point $\mathfrak{p} \in U$; then $\kappa(\mathfrak{p})=\mathbf{k}$ and hence $B \subseteq \mathbf{k}^{[n]}$, proving that $(\mathrm{b}) \Rightarrow(\mathrm{a})$. Implications $(\mathrm{a}) \Rightarrow(\mathrm{d}) \Rightarrow(\mathrm{b})$ are clear and $(c) \Leftrightarrow(b)$ is part of Def. 1.10.

The following example shows that $\mathcal{X}_{\mathbf{k}}(B)$ is not always a constructible subset of $\operatorname{Spec} B$.

1.15. Example. Suppose that $\mathbf{k}$ is a field of characteristic 2 and that $a \in \mathbf{k}$ does not have a square root in $\mathbf{k}$. Define $B=\mathbf{k}[X, Y] /\left(Y^{2}+a X^{2}+X\right)$, where $\mathbf{k}[X, Y]=\mathbf{k}^{[2]}$. We leave it to the reader to check that for any extension field $K$ of $\mathbf{k}$,

$$
K / \mathbf{k} \in \mathrm{PE}_{\mathbf{k}}(B) \Longleftrightarrow K \otimes_{\mathbf{k}} B=K^{[1]} \Longleftrightarrow K \text { contains a square root of } a \text {. }
$$

Consequently, $X_{\mathbf{k}}(B)=\{\mathfrak{p} \in \operatorname{Spec} B \mid \kappa(\mathfrak{p})$ contains a square root of $a\}$. Let us argue that both $X_{\mathbf{k}}(B)$ and $\operatorname{Spec}(B) \backslash X_{\mathbf{k}}(B)$ are infinite sets. 
Given $\lambda \in \mathbf{k},\left(Y^{2}+a X^{2}+X,(X-\lambda)^{2}-a\right)$ is a proper ideal of $\mathbf{k}[X, Y]$ since the equations $Y^{2}+a X^{2}+X=0$ and $(X-\lambda)^{2}=a$ have solutions in the algebraic closure of $\mathbf{k}$. It follows that there exists a maximal ideal $\mathfrak{m}_{\lambda}$ of $B$ such that $(x-\lambda)^{2}-a \in \mathfrak{m}_{\lambda}$, where $x, y \in B$ are the canonical images of $X, Y$. We have $\mathfrak{m}_{\lambda} \in \mathcal{X}_{\mathbf{k}}(B)$, and it is clear that $\lambda \mapsto \mathfrak{m}_{\lambda}$ is injective, so $\mathcal{X}_{\mathbf{k}}(B)$ is an infinite set ( $\mathbf{k}$ is an infinite field, since it is not perfect).

One can see directly that $\operatorname{Frac}(B)=\mathbf{k}(y / x)$, so $B$ is rational over $\mathbf{k}$ and hence $\operatorname{Spec} B$ has infinitely many k-rational points. If $\mathfrak{p} \in \operatorname{Spec} B$ is a $\mathbf{k}$-rational point then $\kappa(\mathfrak{p})=\mathbf{k}$ does not contain a square root of $a$, so $\mathfrak{p} \notin X_{\mathbf{k}}(B)$. So $\operatorname{Spec}(B) \backslash X_{\mathbf{k}}(B)$ is an infinite set. Note in particular that $X_{\mathbf{k}}(B)$ is not a constructible subset of $\operatorname{Spec}(B)$.

One should note that $\operatorname{Spec} B$, in the above example, is a nontrivial form of the affine line over a field of positive characteristic. In characteristic zero, it is not known whether $X_{\mathbf{k}}(B)$ is always a constructible subset of Spec $B 2$ In the following characteristic zero example we are unable to decide whether $X_{\mathbf{k}}(B)$ is constructible, but it seems plausible that it is not.

1.16. Example. Let $\mathbf{k}$ be the field of fractions of the domain $\mathbb{R}[u, v] /\left(u^{2}+v^{2}+1\right)$ (where $\left.\mathbb{R}[u, v]=\mathbb{R}^{[2]}\right)$ and let $B=\mathbf{k}[X, Y, Z] /\left(X^{2}+Y^{2}+Z^{2}+1\right)$ (where $\left.\mathbf{k}[X, Y, Z]=\mathbf{k}^{[3]}\right)$. One can see that $\mathbf{k}$ does not contain a square root of -1 , so $\mathbb{R}$ is algebraically closed in $\mathbf{k}$. We claim:

(a) k-rational points are dense in $\operatorname{Spec} B$;

(b) $X_{\mathbf{k}}(B)$ is dense in $\operatorname{Spec} B$;

(c) $\mathcal{X}_{\mathbf{k}}(B)$ is a constructible subset of $\operatorname{Spec} B \Longleftrightarrow B \subseteq \mathbf{k}^{[2]}$.

To prove (a), observe that given any pair $(a, b) \in \mathbb{R}^{2}$ satisfying $a^{2}+b^{2}>1$ the triple

$$
(x, y, z)=\left(a u+b v, b u-a v, \sqrt{a^{2}+b^{2}-1}\right) \in \mathbf{k}^{3}
$$

satisfies $x^{2}+y^{2}+z^{2}+1=0$ and hence determines a $\mathbf{k}$-rational point of Spec $B$. It can be seen that this collection of points is dense in $\operatorname{Spec} B$, so (a) is true.

(b) Write $\kappa(\mathfrak{p})=B_{\mathfrak{p}} / \mathfrak{p} B_{\mathfrak{p}}$ for each $\mathfrak{p} \in \operatorname{Spec} B$, and note that

$$
\text { if } \kappa(\mathfrak{p}) \text { contains a square root of }-1 \text { then } \mathfrak{p} \in X_{\mathbf{k}}(B) \text {. }
$$

Indeed, if $i \in \kappa(\mathfrak{p})$ satisfies $i^{2}=-1$ and if we define $X_{1}=X+i Y$ and $Y_{1}=X-i Y$ then $\kappa(\mathfrak{p}) \otimes_{\mathbf{k}} B=\kappa(\mathfrak{p})[X, Y, Z] /\left(X^{2}+Y^{2}+Z^{2}+1\right)=\kappa(\mathfrak{p})\left[X_{1}, Y_{1}, Z\right] /\left(X_{1} Y_{1}+Z^{2}+1\right)$ is a "Danielewski surface" and hence $\kappa(\mathfrak{p}) \otimes_{\mathbf{k}} B \subseteq \kappa(\mathfrak{p})^{[2]}$, as is well known. Then $\mathfrak{p} \in X_{\mathbf{k}}(B)$ and (6) is proved. Consider $R=\mathbb{R}[X, Y, Z] /\left(X^{2}+Y^{2}+Z^{2}+1\right)$ and observe that $B=\mathbf{k} \otimes_{\mathbb{R}} R$, so the canonical morphism $f: \operatorname{Spec} B \rightarrow \operatorname{Spec} R$ is an open map by [Sta18, Tag 037G]. Now let $U$ be a nonempty open subset of Spec $B$; then $f(U)$ is a nonempty open subset of Spec $R$, so in particular there exists a maximal ideal $\mathfrak{m}$ of $R$ such that $\mathfrak{m} \in f(U)$. So we may choose $\mathfrak{p} \in U$ such that $f(\mathfrak{p})=\mathfrak{m}$. Since $R / \mathfrak{m} \cong \mathbb{C}$ and $\kappa(\mathfrak{p})$ is an extension of $R / \mathfrak{m}$, it follows that $\kappa(\mathfrak{p})$ contains a square root of -1 and hence that $\mathfrak{p} \in \mathcal{X}_{\mathbf{k}}(B)$, by (6) $)$. This proves $(\mathrm{b})$.

(c) If $X_{\mathbf{k}}(B)$ is constructible then, by $(\mathrm{b}), X_{\mathbf{k}}(B)$ has nonempty interior; by (a) and Rem. 1.14, it follows that $B \subseteq \mathbf{k}^{[2]}$. Conversely, if $B \subseteq \mathbf{k}^{[2]}$ then $\mathcal{X}_{\mathbf{k}}(B)=\operatorname{Spec} B$, so $\mathcal{X}_{\mathbf{k}}(B)$ is constructible.

\footnotetext{
${ }^{2}$ It can be shown that if $\mathbf{k}$ is a field of characteristic zero and $B$ is a 1-dimensional affine $\mathbf{k}$-domain then $X_{\mathbf{k}}(B)$ is either empty or equal to $\operatorname{Spec} B$, so in particular $X_{\mathbf{k}}(B)$ is constructible.
} 
Thus (a), (b) and (c) are true. Because $\mathbf{k}$ does not contain a square root of -1 , we cannot imagine how to embed $B$ in $\mathbf{k}^{[2]}$; in that sense, it seems plausible that $X_{\mathbf{k}}(B)$ is not constructible. However, we don't know if the condition $B \subseteq \mathbf{k}^{[2]}$ is true, so we don't know if $\mathcal{X}_{\mathbf{k}}(B)$ is constructible.

\section{The posets $\mathscr{A}(B)$ AND $\mathscr{K}(B)$}

Paragraph 2.1 states some basic facts about locally nilpotent derivations. For background on this, we refer the reader to any of [vdE00], [Fre06] or [Dai].

2.1. Let $B$ be a domain of characteristic zero.

(i) Let $D \in \operatorname{LND}(B)$ and write $A=\operatorname{ker} D$. Then $A$ is factorially closed in $B$, i.e., the implication $x y \in A \Rightarrow x, y \in A$ is true for all $x, y \in B \backslash\{0\}$. It follows that $A^{*}=B^{*}$ and hence that if $K$ is any field included in $B$ then $K \subseteq A$. Moreover, if $B$ is a UFD then so is $A$.

(ii) Let $D \in \operatorname{LND}(B) \backslash\{0\}$ and write $A=\operatorname{ker} D$. Then there exists $s \in B$ satisfying $D(s) \neq 0$ and $D^{2}(s)=0$, and given any such element $s$ we have $B_{a}=A_{a}[s]=A_{a}^{[1]}$, where we set $a=D(s)$. It follows that if we define $K=$ Frac $A$ then $B \subseteq K[s]=K^{[1]}$ and Frac $B=K^{(1)}$ (in particular $K$ is algebraically closed in Frac $B$ ). Moreover, if $0 \neq b \in B \subseteq K[s]$ then the degree of $b$ as a polynomial in $s$ is equal to the greatest $n \in \mathbb{N}$ satisfying $D^{n}(b) \neq 0$.

We now recall the definition of the posets $\mathscr{A}(B)$ and $\mathscr{K}(B)$, which are invariants of the ring $B$. These objects are defined and studied in [Dai18. In the present paper we are mostly interested in $\mathscr{K}(B)$.

2.2. Let $B$ be a domain of characteristic zero. Given a subset $\Delta$ of $\operatorname{LND}(B)$, define $A_{\Delta}=\bigcap_{D \in \Delta} \operatorname{ker} D$ and $K_{\Delta}=\bigcap_{D \in \Delta} \operatorname{Frac}($ ker $D)$, where the first intersection is taken in $B$ and the second in Frac $B$ (in particular, $A_{\varnothing}=B$ and $K_{\varnothing}=$ Frac $\left.B\right)$. Then define the two sets

$$
\mathscr{A}(B)=\left\{A_{\Delta} \mid \Delta \subseteq \operatorname{LND}(B)\right\} \quad \text { and } \quad \mathscr{K}(B)=\left\{K_{\Delta} \mid \Delta \subseteq \operatorname{LND}(B)\right\} .
$$

Note that $\mathscr{A}(B)$ is a nonempty set of subrings of $B ;(\mathscr{A}(B), \subseteq)$ is a poset, its greatest element is $B$ and its least element is $\operatorname{ML}(B)$. Similarly, $\mathscr{K}(B)$ is a nonempty set of subfields of Frac $B$ whose greatest element is $\operatorname{Frac} B$ and whose least element is $\operatorname{FML}(B)$.

Refer to Section 3 of [Dai18] for the proofs of Lemmas 2.3, 2.4 and 2.7.

2.3. Lemma. If $B$ is a domain of characteristic zero then each element of $\mathscr{A}(B)$ is factorially closed in $B$ and each element of $\mathscr{K}(B)$ is algebraically closed in Frac $B$. In particular, $\operatorname{FML}(B)$ is algebraically closed in Frac $B$.

2.4. Lemma. Let $B$ be a domain of characteristic zero.

(a) Let $D \in \operatorname{LND}(B)$ and let $D^{\prime} \in \operatorname{Der}(\operatorname{Frac} B)$ be the unique extension of $D$ to a derivation of Frac $B$. Then Frac $(\operatorname{ker} D)=\operatorname{ker} D^{\prime}$.

(b) For any subset $\Delta$ of $\operatorname{LND}(B)$, we have $B \cap K_{\Delta}=A_{\Delta}$. Consequently,

$$
\mathscr{K}(B) \rightarrow \mathscr{A}(B), \quad K \mapsto B \cap K,
$$

is a well-defined surjective map and moreover $B \cap \operatorname{FML}(B)=\operatorname{ML}(B)$. 
Recall that if $B$ is a $\mathbb{Q}$-algebra then each $D \in \operatorname{LND}(B)$ determines an automorphism $\exp (D)$ of the ring $B$, defined by $b \mapsto \sum_{i=0}^{\infty} \frac{D^{n}(b)}{n !}$ for $b \in B$. In the case where $B$ is a domain, $\exp (D)$ has a unique extension to an automorphism of Frac $B$.

2.5. Lemma. Let $\mathbf{k}$ be a field of characteristic zero, $B$ a $\mathbf{k}$-domain and $\Delta \subseteq \operatorname{LND}(B)$. Then

$$
K_{\Delta}=\left\{\xi \in \operatorname{Frac} B \mid \forall_{D \in \Delta} E_{D}(\xi)=\xi\right\}=\left\{\xi \in \operatorname{Frac} B \mid \forall_{a \in \mathbf{k}} \forall_{D \in \Delta} E_{a D}(\xi)=\xi\right\},
$$

where for each $D \in \operatorname{LND}(B)$ we let $E_{D} \in \operatorname{Aut}(\operatorname{Frac} B)$ be the unique extension of $\exp (D) \in \operatorname{Aut}(B)$.

Remark. This can be written as $K_{\Delta}=(\operatorname{Frac} B)^{G}=(\operatorname{Frac} B)^{G^{\prime}}$, where $G$ (resp. $\left.G^{\prime}\right)$ denotes the subgroup of $\operatorname{Aut}($ Frac $B)$ generated by $\left\{E_{D} \mid D \in \Delta\right\}$ (resp. by $\left\{E_{a D} \mid a \in \mathbf{k}\right.$ and $\left.D \in \Delta\right\}$ ).

Proof of Lemma 2.5. It suffices to prove that, for each $D \in \operatorname{LND}(B)$,

$$
\operatorname{Frac}(\operatorname{ker} D)=\left\{\xi \in \operatorname{Frac} B \mid E_{D}(\xi)=\xi\right\} .
$$

Moreover, we may assume that $D \neq 0$. Let $A=\operatorname{ker} D, S=A \backslash\{0\}$, and $K=S^{-1} A$. Then $S^{-1} D \in$ $\operatorname{LND}\left(S^{-1} B\right)$ and there exists $t \in S^{-1} B$ such that $\left(S^{-1} D\right)(t)=1$. By 2.1, $S^{-1} B=K[t]=K^{[1]}$ and $S^{-1} D=\frac{d}{d t}$. Now $\exp (D) \in \operatorname{Aut}(B)$ extends to $\exp \left(\frac{d}{d t}\right) \in \operatorname{Aut}(K[t])$, which is the $K$-automorphism of $K[t]$ that sends $t$ to $t+1$. Consequently, we have Frac $B=K(t)$ and $E_{D}: K(t) \rightarrow K(t)$ is the $K$ automorphism that sends $t$ to $t+1$. We leave it as an exercise to check that $\left\{\xi \in K(t) \mid E_{D}(\xi)=\xi\right\}$ is equal to $K$. This proves the Lemma.

Next, we consider how $\mathscr{K}(B)$ behaves under extension of the base field.

2.6. Let $B$ be an algebra over a field $\mathbf{k}$ of characteristic zero and let $D \in \operatorname{LND}(B)$. Let $\overline{\mathbf{k}}$ be any field extension of $\mathbf{k}$ and define $\bar{B}=\overline{\mathbf{k}} \otimes_{\mathbf{k}} B$. Applying the functor $\overline{\mathbf{k}} \otimes_{\mathbf{k}}\left(\_\right): \mathbf{k}$-Mod $\rightarrow \overline{\mathbf{k}}$-Mod to $D$ gives a $\overline{\mathbf{k}}$-linear map $\bar{D}: \bar{B} \rightarrow \bar{B}$, given by $\bar{D}(\lambda \otimes b)=\lambda \otimes D(b)$ for all $\lambda \in \overline{\mathbf{k}}$ and $b \in B$. It is easily verified that $\bar{D} \in \operatorname{LND}(\bar{B})$, so we have a well-defined set map $D \mapsto \bar{D}$ from $\operatorname{LND}(B)$ to $\operatorname{LND}(\bar{B})$. If $D \in \operatorname{LND}(B)$ and $A=\operatorname{ker} D$, then $\operatorname{ker}(\bar{D})=\overline{\mathbf{k}} \otimes_{\mathbf{k}} A$ because $\overline{\mathbf{k}} \otimes_{\mathbf{k}}\left({ }_{-}\right)$is an exact functor.

2.7. Lemma. Let $\overline{\mathbf{k}} / \mathbf{k}$ be an algebraic extension of fields of characteristic zero. Let $B$ be an affine $\mathbf{k}$-domain satisfying $\operatorname{FML}(B)=\mathbf{k}$, and define $\bar{B}=\overline{\mathbf{k}} \otimes_{\mathbf{k}} B$.

(a) $\bar{B}$ is an affine $\overline{\mathbf{k}}$-domain satisfying $\operatorname{FML}(\bar{B})=\overline{\mathbf{k}}$ and $\operatorname{dim}(\bar{B})=\operatorname{dim} B$.

(b) Each $D \in \operatorname{LND}(B)$ has a unique extension $\bar{D} \in \operatorname{LND}(\bar{B})$. Every subset $\Delta$ of $\operatorname{LND}(B)$ determines a subset $\bar{\Delta}$ of $\operatorname{LND}(\bar{B})$ defined by $\bar{\Delta}=\{\bar{D} \mid D \in \Delta\}$. We have

$$
\overline{\mathbf{k}} \otimes_{\mathbf{k}} K_{\Delta}=K_{\bar{\Delta}} \in \mathscr{K}(\bar{B}) \quad \text { for every subset } \Delta \text { of } \operatorname{LND}(B) \text {. }
$$

In particular, for each $K \in \mathscr{K}(B)$ we have $\overline{\mathbf{k}} \otimes_{\mathbf{k}} K \in \mathscr{K}(\bar{B})$.

(c) The map

$$
\begin{aligned}
\mathscr{K}(B) & \rightarrow \mathscr{K}(\bar{B}) \\
K & \mapsto \overline{\mathbf{k}} \otimes_{\mathbf{k}} K
\end{aligned}
$$

is injective and preserves transcendence degree:

$$
\operatorname{trdeg}(\operatorname{Frac}(B): K)=\operatorname{trdeg}\left(\operatorname{Frac}(\bar{B}): \overline{\mathbf{k}} \otimes_{\mathbf{k}} K\right) \quad \text { for all } K \in \mathscr{K}(B) .
$$




\section{Rings HAVING TRIVIAL FML-INVARIANT}

3.1. Definition. Let $\overline{\mathbf{k}}$ be the algebraic closure of a field $\mathbf{k}$. An affine $\mathbf{k}$-domain $B$ is said to be geometrically rational (resp. geometrically unirational) over $\mathbf{k}$ if $\overline{\mathbf{k}} \otimes_{\mathbf{k}} B$ is a domain and the field extension $\operatorname{Frac}\left(\overline{\mathbf{k}} \otimes_{\mathbf{k}} B\right) / \overline{\mathbf{k}}$ is rational (resp. unirational).

The following is a straightforward consequence of the Unirationality Theorem (stated in the introduction) and of Lemma 2.7;

3.2. Corollary. Let $\mathbf{k}$ be a field of characteristic zero and $B$ an affine $\mathbf{k}$-domain satisfying $\operatorname{FML}(B)=\mathbf{k}$. Then $B$ is geometrically unirational over $\mathbf{k}$.

Proof. Consider the algebraic closure $\overline{\mathbf{k}}$ of $\mathbf{k}$. By Lemma 2.7, $\bar{B}=\overline{\mathbf{k}} \otimes_{\mathbf{k}} B$ is an affine $\overline{\mathbf{k}}$-domain satisfying $\operatorname{FML}(\bar{B})=\overline{\mathbf{k}}$. By the Unirationality Theorem, it follows that $\bar{B}$ is unirational over $\overline{\mathbf{k}}$.

Our objective for the rest of this section is to show that if $\operatorname{FML}(B)=\mathbf{k}$ then $B$ satisfies a condition stronger than geometric unirationality. This is achieved in Thm 3.8 (see also Rem. 3.9).

3.3. Notations. Given an algebra $B$ over a field $\mathbf{k}$ of characteristic zero and a finite sequence $\mathcal{S}=\left(D_{1}, \ldots, D_{N}\right)$ of elements of $\operatorname{LND}(B)$ (where $N \geq 1$ and where $D_{1}, \ldots, D_{N}$ are not necessarily distinct), we proceed to define a k-homomorphism $\Psi_{\mathcal{S}}: B \rightarrow B[X]$, where $B[X]$ is the polynomial $\operatorname{ring} B\left[X_{1}, \ldots, X_{N}\right]=B^{[N]}$.

For each $i \in\{1, \ldots, N\}$, let $\delta_{i} \in \operatorname{LND}\left(B\left[X_{1}, \ldots, X_{N}\right]\right)$ be the unique extension of $D_{i}$ such that $\delta_{i}\left(X_{j}\right)=0$ for all $j \in\{1, \ldots, N\}$, note that $X_{i} \delta_{i} \in \operatorname{LND}\left(B\left[X_{1}, \ldots, X_{N}\right]\right)$, and let $\varepsilon_{i}=\exp \left(X_{i} \delta_{i}\right) \in$ $\operatorname{Aut}_{\mathbf{k}}\left(B\left[X_{1}, \ldots, X_{N}\right]\right)$. Define $\Psi_{\mathcal{S}}: B \rightarrow B\left[X_{1}, \ldots, X_{N}\right]$ to be the composition

$$
B \hookrightarrow B\left[X_{1}, \ldots, X_{N}\right] \stackrel{\varepsilon_{N}{ }^{\cdots \cdots} \varepsilon_{1}}{\longrightarrow} B\left[X_{1}, \ldots, X_{N}\right]
$$

and note that $\Psi_{\mathcal{S}}$ is explicitly given by

$$
\Psi_{\mathcal{S}}(b)=\sum_{\left(i_{1}, \ldots, i_{N}\right) \in \mathbb{N}^{N}}\left(\frac{\left(D_{N}^{i_{N}} \circ \cdots \circ D_{1}^{i_{1}}\right)(b)}{i_{1} ! \cdots i_{N} !}\right) X_{1}^{i_{1}} \cdots X_{N}^{i_{N}} \quad \text { for all } b \in B .
$$

By the special case $R=B$ of Notations 1.6, $\Psi_{\mathcal{S}}$ determines for each $\mathfrak{p} \in \operatorname{Spec} B$

- a k-homomorphism $\Psi_{\mathcal{S}}^{\mathfrak{p}}: B \rightarrow \kappa(\mathfrak{p})[X]$

- and a $\kappa(\mathfrak{p})$-homomorphism $\hat{\Psi}_{\mathcal{S}}^{\mathfrak{p}}: \kappa(\mathfrak{p}) \otimes_{\mathbf{k}} B \rightarrow \kappa(\mathfrak{p})[X]$,

where $\kappa(\mathfrak{p})=B_{\mathfrak{p}} / \mathfrak{p} B_{\mathfrak{p}}$ and $\kappa(\mathfrak{p})[X]=\kappa(\mathfrak{p})\left[X_{1}, \ldots, X_{N}\right]=\kappa(\mathfrak{p})^{[N]}$. Let us recall how these homomorphisms are defined. Let $\varphi_{\mathfrak{p}}: B \rightarrow \kappa(\mathfrak{p})$ be the canonical homomorphism and let $\tilde{\varphi}_{\mathfrak{p}}: B[X] \rightarrow \kappa(\mathfrak{p})[X]$ be the unique extension of $\varphi_{\mathfrak{p}}$ satisfying $\tilde{\varphi}_{\mathfrak{p}}\left(X_{i}\right)=X_{i}$ for all $i$. Then let $\Psi_{\mathcal{S}}^{\mathfrak{p}}$ be the composition

$$
B \stackrel{\Psi_{s}}{\longrightarrow} B\left[X_{1}, \ldots, X_{N}\right] \stackrel{\tilde{\varphi}_{\mathfrak{p}}}{\longrightarrow} \kappa(\mathfrak{p})\left[X_{1}, \ldots, X_{N}\right]
$$

and note that

$$
\Psi_{\mathcal{S}}^{\mathfrak{p}}(b)=\sum_{\left(i_{1}, \ldots, i_{N}\right) \in \mathbb{N}^{N}} \varphi_{\mathfrak{p}}\left(\frac{\left(D_{N}^{i_{N}} \circ \cdots \circ D_{1}^{i_{1}}\right)(b)}{i_{1} ! \cdots i_{N} !}\right) X_{1}^{i_{1}} \cdots X_{N}^{i_{N}} \quad \text { for all } b \in B
$$

The $\kappa(\mathfrak{p})$-homomorphism $\hat{\Psi}_{\mathcal{S}}^{\mathfrak{p}}$ is defined via the pushout square (11); it satisfies

$$
\begin{aligned}
\hat{\Psi}_{\mathcal{S}}^{\mathfrak{p}}: \kappa(\mathfrak{p}) \otimes_{\mathbf{k}} B & \longrightarrow \kappa(\mathfrak{p})\left[X_{1}, \ldots, X_{N}\right] . \\
\lambda \otimes b & \longmapsto \lambda \Psi_{\mathcal{S}}^{\mathfrak{p}}(b)
\end{aligned}
$$


3.4. Notations. (1) If $\Psi: R \rightarrow S$ is a ring homomorphism, we write $\Psi^{*}:$ Spec $S \rightarrow$ Spec $R$ for the morphism of schemes determined by $\Psi$.

Let $\mathbf{k}$ be an algebraically closed field of characteristic zero, $B$ an affine $\mathbf{k}$-domain, and $X=\operatorname{Spec} B$.

(2) If $\mathcal{S}=\left(D_{1}, \ldots, D_{N}\right)$ is a finite sequence of elements of $\operatorname{LND}(B)$, we consider the morphism of k-varieties $\Psi_{\mathcal{\delta}}^{*}: \mathbb{A}^{N} \times X \rightarrow X$ determined by the k-homomorphism $\Psi_{\mathcal{s}}: B \rightarrow B\left[X_{1}, \ldots, X_{N}\right]$ defined in 3.3 .

(3) Let $\Delta$ be a subset of $\operatorname{LND}(B)$. For each $\lambda \in \mathbf{k}$ and $D \in \Delta$, we have $\exp (\lambda D) \in \operatorname{Aut}_{\mathbf{k}}(B)$ and hence $\exp (\lambda D)^{*} \in \operatorname{Aut}(X)$. Let $G_{\Delta}$ be the subgroup of $\operatorname{Aut}(X)$ generated by the set $\left\{\exp (\lambda D)^{*} \mid \lambda \in \mathbf{k}, D \in \Delta\right\}$. For each closed point $x \in X$, let $G_{\Delta}(x) \subseteq X$ denote the orbit of $x$ with respect to the natural action of $G_{\Delta}$ on $X$.

3.5. Lemma. Let $\mathbf{k}$ be an algebraically closed field of characteristic zero, $B$ an affine $\mathbf{k}$-domain, and $\Delta$ a subset of $\operatorname{LND}(B)$. Let $X=\operatorname{Spec} B$.

(a) There exists a finite sequence $\mathcal{S}=\left(D_{1}, \ldots, D_{N}\right)$ of elements of $\Delta$ such that the morphism $\Psi_{s}^{*}: \mathbb{A}^{N} \times X \rightarrow X$ has the following property:

For every closed point $x \in X, \Psi_{\mathcal{S}}^{*}\left(\mathbb{A}^{N} \times\{x\}\right)=G_{\Delta}(x)$.

(b) There exists a nonempty Zariski-open subset $U$ of $X$ such that, for every closed point $x \in U$, the dimension of $G_{\Delta}(x)$ is equal to the transcendence degree of Frac $B$ over $K_{\Delta}$.

This result is a corollary of Thm 1, Thm 2 and Cor. 2 of [Pop14. The proof below explains how to use Popov's results to obtain Lemma 3.5. One should read the definitions given on pages 551-552 of [Pop14] before reading this proof.

Proof of Lemma 3.5. If $D \in \Delta$ and $\lambda \in \mathbf{k}$ then $\exp (\lambda D) \in \operatorname{Aut}_{\mathbf{k}}(B)$ and hence $\exp (\lambda D)^{*} \in \operatorname{Aut}(X)$. Let us write $F_{D}=\left\{\exp (\lambda D)^{*}\right\}_{\lambda \in \mathbf{k}}=\left\{\exp (\lambda D)^{*}\right\}_{\lambda \in \mathbb{A}^{1}}$, then $F_{D}$ is a unital algebraic family in $\operatorname{Aut}(X)$ (this is defined on page 551 of Pop14). Thus, $\mathcal{I}_{\Delta}=\left\{F_{D} \mid D \in \Delta\right\}$ is a collection of unital algebraic families in $\operatorname{Aut}(X)$ and $G_{\Delta}$ is generated by $\mathcal{I}_{\Delta}$. It then follows from Lemma 1 of Pop14 that $G_{\Delta}$ is a connected subgroup of $\operatorname{Aut}(X)$ (this notion is defined in the second paragraph of page 552 of [Pop14]).

Note that if $\mathcal{S}=\left(D_{1}, \ldots, D_{N}\right)$ is a finite sequence of elements of $\Delta$ then the morphism $\Psi_{\mathcal{S}}^{*}$ : $\mathbb{A}^{N} \times X \rightarrow X$ is such that $\Psi_{\mathcal{S}}^{*}(t, \ldots) \in \operatorname{Aut}(X)$ for each $t \in \mathbb{A}^{N}$, so if we define $F_{\mathcal{S}}=\left\{\Psi_{\mathcal{S}}^{*}\left(t, Z_{-}\right)\right\}_{t \in \mathbb{A}^{N}}$ then $F_{\mathcal{S}}$ is a unital algebraic family in $\operatorname{Aut}(X)$. Moreover, a moment's reflexion shows that $\left\{F_{\mathcal{S}} \mid\right.$ $\mathcal{S}$ is a finite sequence of elements of $\Delta\}$ is precisely the set of all families in $\operatorname{Aut}(X)$ that are derived from $\mathcal{I}_{\Delta}$ (this concept is defined in the last paragraph of page 551 of [Pop14]).

By Thm 1 of [Pop14], there exists a family derived from $\mathcal{I}_{\Delta}$ and exhaustive for the natural action of $G_{\Delta}$ on $X$ (defined on page 552). This proves assertion (a) of the Lemma.

Note that if we identify the function field $\mathbf{k}(X)$ of $X$ with $\operatorname{Frac}(B)$, then Lemma 2.5 implies that $\mathbf{k}(X)^{G_{\Delta}}=K_{\Delta}$. Thus

$$
\operatorname{trdeg}\left(\operatorname{Frac}(B): K_{\Delta}\right)=\operatorname{trdeg}\left(\mathbf{k}(X): \mathbf{k}(X)^{G_{\Delta}}\right) .
$$

So we may apply Thm 2 and Cor. 2 of Pop14 as follows:

(i) By Thm 2, there exist an $m \in \mathbb{N}$ and a nonempty open subset $U$ of $X$ such that, for every closed point $x \in U$, the dimension of $G_{\Delta}(x)$ is equal to $m$. 
(ii) By Cor. 2, the transcendence degree of $\mathbf{k}(X)^{G_{\Delta}}$ over $\mathbf{k}$ is equal to $\operatorname{dim} X-m$, where $m$ is the same as in (i).

This gives $m=\operatorname{dim} X-\operatorname{trdeg}\left(\mathbf{k}(X)^{G_{\Delta}}: \mathbf{k}\right)=\operatorname{trdeg}\left(\mathbf{k}(X): \mathbf{k}(X)^{G_{\Delta}}\right)$, which is equal to $\operatorname{trdeg}(\operatorname{Frac}(B)$ : $\left.K_{\Delta}\right)$ by (9). This proves assertion (b) of the Lemma.

The following is an intermediate result that will be improved in Thm 3.8 :

3.6. Lemma. Let $\mathbf{k}$ be a field of characteristic zero and $B$ an affine $\mathbf{k}$-domain satisfying $\mathrm{FML}(B)=$ k. Let $\Delta$ be any subset of $\operatorname{LND}(B)$ satisfying $K_{\Delta}=\mathbf{k}$. Then there exist a finite sequence $\mathcal{S}=$ $\left(D_{1}, \ldots, D_{N}\right)$ of elements of $\Delta$ and a nonempty Zariski-open subset $U$ of Spec $B$ such that, for each maximal ideal $\mathfrak{m}$ of $B$ belonging to $U$, the $\mathbf{k}$-homomorphism $\Psi_{\mathcal{S}}^{\mathfrak{m}}: B \rightarrow \kappa(\mathfrak{m})\left[X_{1}, \ldots, X_{N}\right]$ is injective.

Proof. Let us first prove the Lemma under the additional assumption that $\mathbf{k}$ is algebraically closed. Consider any subset $\Delta$ of $\operatorname{LND}(B)$ satisfying $K_{\Delta}=\mathbf{k}$. By Lemma 3.5, there exists a finite sequence $\mathcal{S}=\left(D_{1}, \ldots, D_{N}\right)$ of elements of $\Delta$ such that the morphism $\Psi_{\mathcal{S}}^{*}: \mathbb{A}^{N} \times X \rightarrow X$ (where $X=\operatorname{Spec} B$ ) satisfies $\Psi_{\mathcal{S}}^{*}\left(\mathbb{A}^{N} \times\{x\}\right)=G_{\Delta}(x)$ for every closed point $x \in X$. Choose such a sequence $\mathcal{S}$. Lemma 3.5 also implies that there exists a nonempty open subset $U$ of Spec $B$ such that, for every closed point $x \in U$, the dimension of $G_{\Delta}(x)$ is equal to the transcendence degree of Frac $B$ over $K_{\Delta}=\mathbf{k}$. Choose such a $U$ and note that if $x$ is a closed point of $U$ then $G_{\Delta}(x)$ is dense in $\operatorname{Spec} B$. This means that if $x$ is a closed point of $U$ then the composition

$$
\begin{aligned}
\mathbb{A}^{N} & \rightarrow \mathbb{A}^{N} \times X \stackrel{\Psi_{s}^{*}}{\longrightarrow} X \\
t & \mapsto(t, x)
\end{aligned}
$$

is a dominant morphism. Now this composition is precisely $\left(\Psi_{\mathcal{S}}^{\mathfrak{m}}\right)^{*}$, where $\mathfrak{m}=x$, so $\Psi_{\mathcal{S}}^{\mathfrak{m}}$ is injective (and this is true for every maximal ideal $\mathfrak{m}$ of $B$ such that $\mathfrak{m} \in U$ ). This proves the Lemma when $\mathbf{k}$ is algebraically closed.

Now drop the assumption that $\mathbf{k}$ is algebraically closed. Consider any subset $\Delta$ of $\operatorname{LND}(B)$ satisfying $K_{\Delta}=\mathbf{k}$. Let $\overline{\mathbf{k}}$ be the algebraic closure of $\mathbf{k}$ and let $\bar{B}=\overline{\mathbf{k}} \otimes_{\mathbf{k}} B$. By Lemma 2.7, $\bar{B}$ is an affine $\overline{\mathbf{k}}$-domain satisfying $\operatorname{FML}(\bar{B})=\overline{\mathbf{k}}$. Define $\bar{\Delta} \subseteq \operatorname{LND}(\bar{B})$ as in Lemma 2.7, then (by Lemma 2.7) $K_{\bar{\Delta}}=\overline{\mathbf{k}} \otimes_{\mathbf{k}} K_{\Delta}=\overline{\mathbf{k}} \otimes_{\mathbf{k}} \mathbf{k}$, so $K_{\bar{\Delta}}=\overline{\mathbf{k}}$. Since the Theorem is valid for $\bar{B}$ by the first part of the proof, there exist a finite sequence $\overline{\mathcal{S}}=\left(\bar{D}_{1}, \ldots, \bar{D}_{N}\right)$ of elements of $\bar{\Delta}$ and a dense open subset $\bar{U}$ of Spec $\bar{B}$ such that, for each maximal ideal $\overline{\mathfrak{m}}$ of $\bar{B}$ belonging to $\bar{U}, \Psi_{\overline{\mathrm{m}}}^{\overline{\mathrm{m}}}: \bar{B} \rightarrow \kappa(\overline{\mathfrak{m}})\left[X_{1}, \ldots, X_{N}\right]$ is injective. Let $\mathcal{S}=\left(D_{1}, \ldots, D_{N}\right)$ be the sequence of elements of $\Delta$ such that $\bar{D}_{i}$ is the extension of $D_{i}$ for all $i$. Let $U$ be the image of $\bar{U}$ by the open map $\operatorname{Spec} \bar{B} \rightarrow \operatorname{Spec} B$ (by [Sta18, Tag 037G], if $R$ and $S$ are algebras over a field $k$ then $\operatorname{Spec}\left(R \otimes_{k} S\right) \rightarrow \operatorname{Spec} R$ is an open morphism). Then $U$ is a dense open subset of Spec $B$ and we claim that $\mathcal{S}$ and $U$ have the desired property. Indeed, let $\mathfrak{m}$ be any maximal ideal of $B$ satisfying $\mathfrak{m} \in U$. Then there exists $\overline{\mathfrak{m}} \in \bar{U}$ satisfying $\overline{\mathfrak{m}} \cap B=\mathfrak{m}$, and $\overline{\mathfrak{m}}$ is in fact a maximal ideal of $\bar{B}$ because $\bar{B}$ is integral over $B$. It is clear from equations (7) and (8) that the diagram

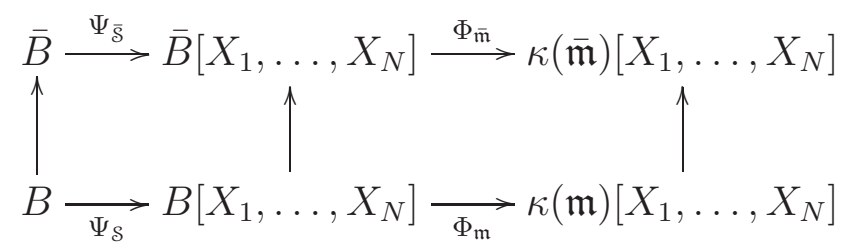


commutes. Since $B \rightarrow \bar{B}$ and $\Psi_{\overline{\mathcal{s}}}^{\overline{\mathrm{m}}}=\Phi_{\overline{\mathfrak{m}}} \circ \Psi_{\overline{\mathcal{s}}}$ are injective, it follows that $\Psi_{\mathcal{S}}^{\mathfrak{m}}=\Phi_{\mathfrak{m}} \circ \Psi_{\mathcal{S}}$ is injective. This completes the proof of Lemma 3.6.

The following well-known fact is very useful:

3.7. Lemma. Let $\mathbf{k}$ be a field of characteristic zero and $B$ a $\mathbf{k}$-domain. The following are equivalent:

(a) $\mathbf{k}$ is algebraically closed in $\operatorname{Frac}(B)$

(b) $K \otimes_{\mathbf{k}} B$ is a domain for every extension field $K$ of $\mathbf{k}$ (i.e., $B$ is geometrically integral).

(c) $\overline{\mathbf{k}} \otimes_{\mathbf{k}} B$ is a domain, where $\overline{\mathbf{k}}$ is the algebraic closure of $\mathbf{k}$.

See 1.12 for the notation $X_{\mathbf{k}}(B)$.

3.8. Theorem. Let $\mathbf{k}$ be a field of characteristic zero and $B$ an affine $\mathbf{k}$-domain. If $\operatorname{FML}(B)=\mathbf{k}$ then the following hold.

(a) The set $X_{\mathbf{k}}(B)$ has nonempty interior, i.e., the condition $\kappa(\mathfrak{p}) \otimes_{\mathbf{k}} B \subseteq \kappa(\mathfrak{p})^{[\operatorname{dim} B]}$ is satisfied for all prime ideals $\mathfrak{p}$ in some nonempty open subset of Spec $B$.

(b) Given any subset $\Delta$ of $\operatorname{LND}(B)$ satisfying $K_{\Delta}=\mathbf{k}$, there exist a finite sequence $\mathcal{S}=$ $\left(D_{1}, \ldots, D_{N}\right)$ of elements of $\Delta$ and a nonempty Zariski-open subset $U$ of Spec $B$ such that, for each $\mathfrak{p} \in U$, the $\kappa(\mathfrak{p})$-homomorphism $\hat{\Psi}_{\mathcal{s}}^{\mathfrak{p}}: \kappa(\mathfrak{p}) \otimes_{\mathbf{k}} B \longrightarrow \kappa(\mathfrak{p})\left[X_{1}, \ldots, X_{N}\right]$ is injective.

Proof. The condition $\operatorname{FML}(B)=\mathbf{k}$ implies that $\mathbf{k}$ is algebraically closed in Frac $B$ by Lemma 2.3, so $B$ is geometrically integral by Lemma 3.7. We first prove (b). Let $\Delta$ be a subset of $\operatorname{LND}(B)$ satisfying $K_{\Delta}=\mathbf{k}$. By Lemma 3.6, there exist a finite sequence $\mathcal{S}=\left(D_{1}, \ldots, D_{N}\right)$ of elements of $\Delta$ and a nonempty Zariski-open subset $U$ of Spec $B$ such that, for each maximal ideal $\mathfrak{m}$ of $B$ belonging to $U$, the $\mathbf{k}$-homomorphism $\Psi_{s}^{\mathfrak{m}}: B \rightarrow \kappa(\mathfrak{m})\left[X_{1}, \ldots, X_{N}\right]$ is injective. Since $B$ is geometrically integral, part (b) of Thm 1.7 implies that $\mathfrak{m} \in \mathcal{X}_{\mathbf{k}}\left(\Psi_{\mathcal{S}}\right)$ for each maximal ideal $\mathfrak{m}$ of $B$ belonging to $U$, so in particular $X_{\mathbf{k}}\left(\Psi_{\mathcal{S}}\right)$ is dense in Spec $B$. Since $X_{\mathbf{k}}\left(\Psi_{\mathcal{S}}\right)$ is a constructible subset of Spec $B$ by part (国) of Thm 1.7, some 3 nonempty open subset of $\operatorname{Spec} B$ is included in $X_{\mathbf{k}}\left(\Psi_{\mathfrak{s}}\right)$, so (b) follows. Since $X_{\mathbf{k}}\left(\Psi_{s}\right) \subseteq X_{\mathbf{k}}(B)$, (a) follows.

3.9. Remarks. Given a field $\mathbf{k}$ and an affine $\mathbf{k}$-domain $B$, consider the conditions

(i) $X_{\mathbf{k}}(B)$ has nonempty interior;

(ii) $\overline{\mathbf{k}} \otimes_{\mathbf{k}} B \subseteq \overline{\mathbf{k}}^{[\operatorname{dim} B]}$, where $\overline{\mathbf{k}}$ is the algebraic closure of $\mathbf{k}$;

(iii) $B$ is geometrically unirational.

Then (i) is strictly stronger than (ii) by Cor. 1.9 and Ex. 1.15, and it is clear that (ii) is strictly stronger than (iii). This justifies our claim that Thm 3.8 goes beyond Cor. 3.2 .

3.10. Corollary. Let $\mathbf{k}$ be a field of characteristic zero and let $B$ be an affine $\mathbf{k}$-domain satisfying $\operatorname{FML}(B)=\mathbf{k}$. Then the following are equivalent:
(a) $B \subseteq \mathbf{k}^{[\operatorname{dim} B]}$
(b) $\mathbf{k}$-rational points are dense in $\operatorname{Spec} B$
(c) the extension $\operatorname{Frac}(B) / \mathbf{k}$ has the density property
(d) $B$ is unirational over $\mathbf{k}$.

\footnotetext{
${ }^{3}$ Actually, one can show that $U$ itself is included in $X_{\mathbf{k}}\left(\Psi_{\mathcal{S}}\right)$.
} 
In particular, if $\mathbf{k}$ is algebraically closed or $\operatorname{dim} B \leq 2$ then $B \subseteq \mathbf{k}^{[\operatorname{dim} B]}$.

Proof. Conditions (a-d) are equivalent by Rem. 1.14, If $\mathbf{k}$ is algebraically closed then (b) holds, so $B \subseteq \mathbf{k}^{[\operatorname{dim} B]}$. If $\operatorname{dim} B \leq 2$ then it is well known that $B$ is rational over $\mathbf{k}$ (see for instance [Kol10, Lemma 5.3.8] or [Dai18, Cor. 2.6]), so (d) holds and hence $B \subseteq \mathbf{k}^{[\operatorname{dim} B]}$.

Question. Let $\mathbf{k}$ be a field of characteristic zero and $B$ an affine $\mathbf{k}$-domain. When $\mathbf{k}$ is not algebraically closed, does the condition $\operatorname{FML}(B)=\mathbf{k}$ imply that $B$ is unirational over $\mathbf{k}$ ? (Probably not, but an example would be welcome.)

\section{REFERENCES}

[AFK $\left.{ }^{+} 13\right]$ I. Arzhantsev, H. Flenner, S. Kaliman, F. Kutzschebauch, and M. Zaidenberg. Flexible varieties and automorphism groups. Duke Math. J., 162(4):767-823, 2013.

[Dai] D. Daigle. Introduction to locally nilpotent derivations. Informal lecture notes prepared in 2010, available at http://aix1.uottawa.ca/ ddaigle

[Dai18] D. Daigle. Locally nilpotent derivations and the structure of rings. preprint, 2018.

[Eak72] P. Eakin. A note on finite dimensional subrings of polynomial rings. Proc. Amer. Math. Soc., 31:75-80, 1972.

[Fre06] G. Freudenburg. Algebraic Theory of Locally nilpotent derivations. Invariant theory and algebraic transformation groups VII. Springer-Verlag, 2006.

[Kol10] R. Kolhatkar. Homogeneous locally nilpotent derivations and affine ML-surfaces. PhD thesis, University of Ottawa, 2010.

[Lie10] A. Liendo. $\mathbb{G}_{a}$-actions of fiber type on affine $\mathbb{T}$-varieties. J. Algebra, 324:3653-3665, 2010.

[Mat80] H. Matsumura. Commutative Algebra. Mathematics Lecture Note Series. Benjamin/Cummings, second edition, 1980.

[Pop11] V. L. Popov. On the Makar-Limanov, Derksen invariants, and finite automorphism groups of algebraic varieties. In Affine algebraic geometry, volume 54 of CRM Proc. Lecture Notes, pages 289-311. Amer. Math. Soc., Providence, RI, 2011.

[Pop13] V. L. Popov. Rationality and the FML invariant. J. Ramanujan Math. Soc., 28A:409-415, 2013.

[Pop14] V. L. Popov. On infinite dimensional algebraic transformation groups. Transform. Groups, 19:549-568, 2014.

[Sta18] The Stacks Project Authors. Stacks Project. http://stacks.math.columbia.edu, 2018.

[vdE00] A. van den Essen. Polynomial automorphisms, volume 190 of Progress in Mathematics. Birkhäuser, 2000.

Department of Mathematics and Statistics, University of Ottawa, Ottawa, Canada K1N 6N5

E-mail address: ddaigle@uottawa.ca 